\title{
The Possibility of Urinary Liver-Type Fatty Acid-Binding Protein as a Biomarker of Renal Hypoxia in Spontaneously Diabetic Torii Fatty Rats
}

\author{
Jun Tanabe ${ }^{a}$ Yuji Ogurab ${ }^{b}$ Mikie Nakabayashic ${ }^{c}$ Yoshio Nagaid

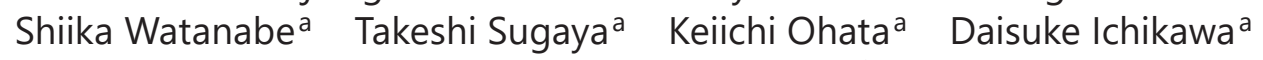 \\ Kazuho Inoue $^{e}$ Seiko Hoshino ${ }^{e}$ Kenjiro Kimura ${ }^{f}$ Yugo Shibagaki ${ }^{a}$ \\ Yumie Ono ${ }^{c}$ Atsuko Kamijo-Ikemoria, e \\ ${ }^{a}$ Division of Nephrology and Hypertension, Department of Internal Medicine, St. \\ Marianna University School of Medicine, Kanagawa, Japan; ${ }^{b}$ Department of Physiology, \\ St. Marianna University School of Medicine, Kanagawa, Japan; ' $D$ Department of Electronics \\ and Bioinformatics, School of Science and Technology, Meiji University, Kanagawa, Japan; \\ ${ }^{\mathrm{d} D i v i s i o n}$ of Metabolism and Endocrinology, Department of Internal Medicine, St. Marianna

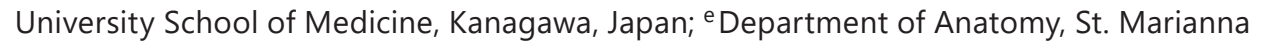

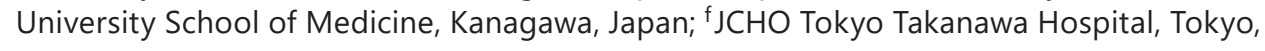 \\ Japan
}

\section{Keywords}

Diabetes · Diabetic kidney disease · Hypoxia - Kidney · Liver-type fatty acid-binding protein · Tubulointerstitial damage

\begin{abstract}
Background: Renal hypoxia is an aggravating factor for tubulointerstitial damage, which is strongly associated with renal prognosis in diabetic kidney disease (DKD). Therefore, urinary markers that can detect renal hypoxia are useful for monitoring DKD. Objective: To determine the correlation between urinary liver-type fatty acid-binding protein (L-FABP) and renal hypoxia using a novel animal model of type 2 diabetes. Methods: Male spontaneously diabetic Torii (SDT) fatty rats $(n=6)$ were used as an animal model of type 2 diabetes. Age- and sexmatched Sprague-Dawley (SD) rats $(n=8)$ were used as controls. Body weight, systolic blood pressure, and blood glucose levels were measured at $8,12,16$, and 24 weeks of age. Urine samples and serum and kidney tissues were collected at 24 weeks of age. Microvascular blood flow index $(\mathrm{BFI})$ was measured using diffuse correlation spectroscopy before sampling both the serum and kidneys for the evaluation of renal microcirculation at the corticomedullary junction. Results: Obesity, hyperglycemia, and hypertension were observed in the SDT fatty
\end{abstract}


rats. Focal glomerular sclerosis, moderate interstitial inflammation, and fibrosis were significantly more frequent in SDT fatty rats than in SD rats. While the frequency of peritubular endothelial cells and phosphoendothelial nitric oxide synthase levels were similar in both types of rats, the degree of renal hypoxia-inducible factor- $1 \alpha$ (HIF-1 $\alpha$ ) expression was significantly higher (and with no change in renal vascular endothelial growth factor expression levels) in the SDT fatty rats. Urinary L-FABP levels were significantly higher and renal microvascular BFI was significantly lower in the SDT fatty rats than in the SD rats. Urinary L-FABP levels exhibited a significant positive correlation with renal HIF-1 $\alpha$ expression and a significant negative correlation with renal microvascular BFI. Conclusions: Urinary L-FABP levels reflect the degree of renal hypoxia in DKD in a type 2 diabetic animal model. Urinary L-FABP may thus prove useful as a renal hypoxia marker for monitoring DKD in patients with type 2 diabetes in clinical practice.

\section{Introduction}

Despite the development of numerous therapeutics for diabetes, diabetic kidney disease (DKD) remains a major cause of end-stage renal failure globally [1,2] and is associated with an increase in medical care expenses [3]. Owing to the limited number of therapeutic strategies presently available, monitoring for DKD is critical for prompt multidisciplinary management, and novel biomarkers that can provide detailed kidney status of patients with DKD are sorely required.

Tubulointerstitial damage is strongly associated with renal prognosis in DKD [4-6], and renal hypoxia is an aggravating factor for the progression of this damage [7-9]. Tubular biomarkers that reflect the degree of renal hypoxia as well as tubulointerstitial damage would therefore be useful for monitoring DKD.

Urinary liver-type fatty acid-binding protein (L-FABP) is a tubular marker that accurately reflects the severity of tubulointerstitial damage in chronic kidney disease including DKD $[10-12]$ and acute kidney disease $[13,14]$. L-FABP has been promulgated as a tubular biomarker by the Ministry of Health, Labour and Welfare in Japan [15]. An increase in urinary L-FABP has been reported to be an independent risk factor for DKD progression in type 1 and 2 diabetes [16-18]. Although urinary L-FABP levels were reported to be associated with renal microcirculation in donors during surgery for renal transplantation [19], no correlation with renal hypoxia in DKD has been elucidated to date. Therefore, we assessed the correlation between urinary L-FABP levels and renal hypoxia in a rat model of type 2 diabetes using spontaneously diabetic Torii (SDT) fatty (SDT.Cg-Lepr ${ }^{\mathrm{fa}} / \mathrm{JttJ}$ (l) rats, a novel model that mimics the human pathophysiology of type 2 diabetes that is accompanied by obesity, hyperlipidemia, and hypertension [20]. The renal hypoxia was evaluated by renal hypoxia-inducible factor- $1 \alpha$ (HIF-1 $\alpha$ ) expression, and the microvascular blood flow index (BFI) was measured using diffuse correlation spectroscopy (DCS).

\section{Materials and Methods}

\section{Animals}

All animal studies were conducted in accordance with the St. Marianna University School of Medicine Institutional Guide for Animal Experiments and the Guide for the Care and Use of Laboratory Animals. Male SDT fatty rats derived from a Sprague-Dawley (SD) colony were used as a model of type 2 diabetes. Four-week-old male SDT fatty rats $(n=7)$ and age-matched 


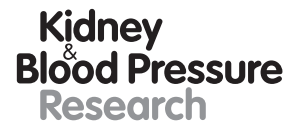

Kidney
Blood Pressure
Research

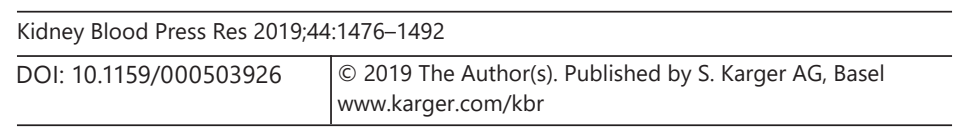

Tanabe et al.: Urinary L-FABP and Renal Hypoxia

control SD rats $(n=8)$ were purchased from CLEA Japan (Tokyo, Japan) and allowed free access to laboratory chow (CRF-2; Charles River Laboratories Japan, Yokohama, Japan) and water. One STD fatty rat died at 23 weeks of age, but the remaining 6 underwent extensive analyses. To confirm the diabetic status, blood glucose levels were measured $4 \mathrm{~h}$ after the removal of the chow with the Free Style Freedom Lite (Niporo, Osaka, Japan) in blood collected from the tail vein.

For all experiments, 24-week-old rats were individually housed overnight in metabolic cages with free access to tap water. Urine was collected for the measurement of urinary markers. Additionally, under inhalation anesthesia using $2 \%$ isoflurane, serum samples and kidney tissues were collected. The extracted kidneys were weighed, sectioned, and stored either at $-80^{\circ} \mathrm{C}$ or in liquid nitrogen for downstream analyses.

\section{Body Weight and Blood Pressure Measurement}

Body weight and systolic blood pressure (SBP) were measured every 4 weeks from 8 to 24 weeks of age. SBP was measured using a tail-cuff apparatus (Softron BP-98A; Softron, Tokyo, Japan), and averages of 3 SBP measurements per animal per time point were recorded [11].

\section{Determination of Microvascular BFI Using DCS}

Microvascular BFI was measured using DCS, which detects tissue blood flow velocity using intensity fluctuations of a reflected near-infrared light due to the movement of red blood cells in the microvasculature [21-23]. The measurements were performed immediately before sampling both the serum and kidneys at 24 weeks of age. Briefly, under inhalation anesthesia using $2 \%$ isoflurane, the rats underwent left abdominal laparotomy. Photonemitter and detector probes were placed at 4-mm intervals directly on the left renal surface to detect the emitted near-infrared light from the renal parenchyma for $2 \mathrm{~min}$. The DCS system enables BFI measurements of the region in approximately half the depth $(2 \mathrm{~mm})$ of the distance between the 2 probes below the renal surface [24]; this allows the detection of microvascular blood flow in the corticomedullary area. The near-infrared light sent from the photon-emitter probe is irradiated from the renal surface to the blood flowing in the peritubular capillaries of the renal cortex. The light is diffused by the red blood cells, and this reflected light is recorded by the detector probe. Microvascular BFI is defined as the blood velocity calculated from an autocorrelation-related change in the reflected light [21] and we used it to determine the degree of change in microvascular blood flow.

\section{Serum and Urinary Biochemistry}

Serum and urine creatinine levels were measured using a Quantichrom ${ }^{\mathrm{TM}}$ creatinine assay kit (BioAssay Systems, Hayward, CA, USA). Serum cystatin C levels were measured using a rat cystatin $\mathrm{C}$ enzyme-linked immunosorbent assay (ELISA) kit (BioVendor, Brno, Czech Republic). Serum urea nitrogen levels were measured via urease-LED-ultraviolet absorption spectrophotometry, serum total cholesterol levels using the cholesterol oxidase enzymatic method, and serum triglyceride levels using an enzymatic method provided by the SRL clinical laboratory testing services (Tokyo, Japan). Urinary glucose levels were determined semiquantitatively using urinary dipsticks (WAKO Pure Chemical Industries, Osaka, Japan). Grading for urinary glucose was as follows: 0, negative; $1+,>100 \mathrm{mg} / \mathrm{dL} ; 2+,>250 \mathrm{mg} /$ $\mathrm{dL} ; 3+,>500 \mathrm{mg} / \mathrm{dL}$; and 4+, >2,000 mg/dL. Levels of urinary L-FABP and albumin were measured using rat L-FABP (CMIC, Tokyo, Japan) and rat albumin (Fujifilm WAKO Shibayagi, Gunma, Japan) ELISA kits, respectively. They were reported as ratios relative to the urinary creatinine levels. 


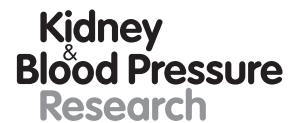

Kidney
Blood Pressure
Research \begin{tabular}{l|l}
\hline Kidney Blood Press Res 2019;44:1476-1492 \\
\hline DOI: 10.1159/000503926 & $\begin{array}{l}\text { @ 2019 The Author(s). Published by S. Karger AG, Basel } \\
\text { www.karger.com/kbr }\end{array}$ \\
\hline
\end{tabular}

Tanabe et al.: Urinary L-FABP and Renal Hypoxia

\section{Renal Histological and Morphometric Analyses}

The midsection of each excised kidney was dissected in the minor axial direction. The 2 pieces were fixed in $10 \%$ buffered formalin or methyl Carnoy's solution (60\% methanol, $30 \%$ chloroform, and 10\% glacial acetic acid) and embedded in paraffin. Serial sections (3 $\mu \mathrm{m}$ thick) were prepared for renal histological assessment including periodic acid-Schiff (PAS) staining and immunohistochemistry (IHC).

Glomerulosclerosis in PAS-stained sections was evaluated by grading the extent of sclerosis in each glomerulus as follows: 0 , no sclerosis; 1 , sclerosis in 1-25\% of the glomerulus; 2 , sclerosis in $26-50 \%$ of the glomerulus; 3 , sclerosis in $51-75 \%$ of the glomerulus; and 4 , sclerosis in $76-100 \%$ of the glomerulus. At $\times 100$ magnification, the glomerulosclerosis score for each animal was calculated as follows: ([1 $\times$ the number of grade 1 glomeruli, \%] $+[2 \times$ the number of grade 2 glomeruli, \%] + [3 $\times$ the number of grade 3 glomeruli, \%] + [4 $\times$ the number of grade 4 glomeruli, \%]). We examined 50 glomeruli for each animal [25].

\section{IHC Analyses of the Renal Tissues}

Target antigens in the preprocessed sections were stained using the indirect immunoperoxidase method, as described previously [11]. Briefly, the formalin-fixed/paraffinembedded tissue specimens were used to stain for myofibroblasts and tubular cells exhibiting the epithelial-mesenchymal transition by using a mouse monoclonal antibody specific for $\alpha$-smooth muscle actin ( $\alpha$-SMA, 1:800; Sigma-Aldrich, St. Louis, MO, USA). The tissue specimens fixed in methyl Carnoy's solution were assessed immunohistochemically for macrophages using a mouse monoclonal antibody (ED-1) specific for CD68 (1:100; Abcam, Tokyo, Japan), for type I and type III collagens using goat polyclonal antibodies specific for type I (1:200; Southern Biotech, Birmingham, AL, USA) and type III (1:200; Southern Biotech) collagen, and for endothelial cells using a mouse monoclonal antibody specific for rat endothelial cell antigen-1 (RECA-1) (1:100; Abcam). Labeled proteins were visualized using polymeric horseradish peroxidase (HRP)-conjugated secondary antibodies (ImmPRESS ${ }^{\mathrm{TM}}$ polymer detection kit; Vector Laboratories, Burlingame, CA, USA). Peroxidase activity was detected via the diaminobenzidine reaction (Liquid DAB+; DAKO Japan, Tokyo, Japan), and sections were counterstained with hematoxylin.

For quantification, images from 10 nonoverlapping fields throughout the cortical and outer medullary regions were captured at $\times 100$ magnification. The extent of macrophage and interstitial myofibroblast infiltration in the cortical and outer medullary interstitia were automatically measured using an image analyzer (WinRoof v6.4, Mitani, Tokyo, Japan). Briefly, the areas positively stained for $\alpha$-SMA, CD68, and RECA-1 were independently measured and expressed as ratios relative to the areas of the entire cortical and outer medullary regions. The same method was used to measure the expression levels of both type I and type III collagen. Furthermore, to evaluate the expansion of mesangial matrix, type IV collagen was labeled using a rabbit polyclonal antibody (1:500; Cedarlane Laboratories, ON, Canada) in sections that were fixed in formalin [26]. Thirty glomeruli in the cortical area were analyzed. Briefly, areas positively stained for type IV collagen in each glomerulus as well as the size of each glomerulus were measured with WinRoof image analyzer, and then expressed as ratios relative to the total glomerular areas.

\section{Real-Time Quantitative Reverse Transcription Polymerase Chain Reaction}

Total RNA was extracted from the kidney tissues using a RNeasy midi kit (Qiagen, Valencia, CA, USA) according to the manufacturer's instructions, and a $0.5-\mu \mathrm{g}$ aliquot was reverse-transcribed. TaqMan real-time polymerase chain reaction (PCR) with StepOnePlus $^{\text {TM }}$ real-time PCR system (Applied Biosystems, Waltham, MA, USA) was used to measure the mRNA levels of monocyte chemoattractant protein $(M c p-1)$, transforming growth 


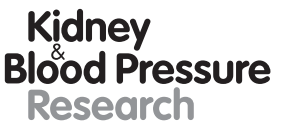

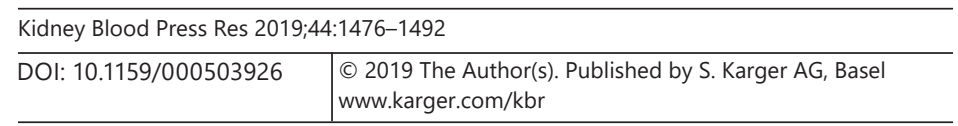

Tanabe et al.: Urinary L-FABP and Renal Hypoxia

factor- $\beta$ (Tgf- $\beta$ ), CD31, Hif- $1 \alpha$, vascular endothelial growth factor (Vegf), and $18 \mathrm{~S}$ ribosomal RNA (18s). The expression levels of all mRNAs were normalized to the levels of $18 \mathrm{~s}$ in all samples.

\section{Renal Protein Expression of Vascular Endothelial Growth Factor}

Renal vascular endothelial growth factor (VEGF) protein expression levels were determined using an ELISA kit from R\&D Systems (Minneapolis, MN, USA) with protein extracted from frozen kidney tissues, after determining protein concentrations as described previously [27].

\section{Western Blotting}

Proteins were extracted from frozen kidney tissues, and protein concentrations were measured as described previously [28]. Next, $60 \mu \mathrm{g}$ of extracts were separated by SDS-PAGE using NuPAGE 4-12\% Bis-Tris gels and the XCell SureLock Mini-Cell system (Thermo Fisher Scientific, Rockford, IL, USA). The separated proteins were transferred to PVD membranes using the iBlot ${ }^{\mathrm{TM}}$ dry blotting system (Thermo Fisher Scientific), and the membranes were blocked in Blocking One solution (Nacalai Tesque, Kyoto, Japan). Primary antibodies against HIF-1 $\alpha$ (rabbit monoclonal, \#14179, 1:1,000; Cell Signaling Technology, Danvers, MA, USA), endothelial nitric oxide synthase (eNOS, rabbit polyclonal, \#9572, 1:1,000; Cell Signaling Technology), and phospho-eNOS (rabbit polyclonal, \#9571, 1:1,000; Cell Signaling Technology) diluted in Can Get Signal Solution I (TOYOBO, Osaka, Japan) were incubated overnight at $4{ }^{\circ} \mathrm{C}$. After washing, the membranes were incubated with an HRP-conjugated anti-rabbit antibody (Santa Cruz Biotechnology, Dallas, TX, USA) diluted at 1:2,000 for phospho-eNOS and eNOS or 1:4,000 for HIF-1 $\alpha$ in Blocking One solution for $1 \mathrm{~h}$ at room temperature. Subsequently, chemiluminescence was detected using ECL Prime Western blotting detection reagent (GE Healthcare, Little Chalfont, UK) with a CCD camera system (Ez-Capture II, ATTO, Tokyo, Japan). A rabbit monoclonal antibody to $\alpha$-tubulin $(1: 4,000 ;$ Abcam) was also used to detect $\alpha$-tubulin on the same membranes that were incubated with HIF- $1 \alpha$, eNOS, and phospho-eNOS, after treatment with a stripping buffer (WAKO); the expression levels of the 3 proteins were normalized to that of $\alpha$-tubulin. The expression levels of all proteins were quantified using ImageJ software (National Institutes of Health, Frederick, MD, USA).

\section{Statistical Analysis}

All values were expressed as means \pm standard error of the mean (SEM). A $p$ value $<0.05$ was considered to indicate statistical significance. Differences between SD and SDT rats were analyzed by the Steel test. The 2 groups were compared using the Mann-Whitney U test. Spearman's rank correlation coefficient was used to evaluate nonparametric data and assess correlations between 2 parameters. All statistical analyses were performed using JMP ${ }^{\circledR}$ software v13.0.0 (SAS Institute, Cary, NC, USA).

\section{Results}

\section{Changes in Body Weight, Blood Glucose, and SBP}

In both groups, the body weight, blood glucose, and SBP values were measured every 4 weeks in rats aged between 8 and 24 weeks (Table 1). Body weight was significantly higher in the 12-, 16-, 20-, and 24-week-old rats than in the 8-week-old rats in both groups. Additionally, the body weights of the 8-, 12-, and 16-week-old SDT fatty rats were significantly higher than those of the age-matched SD rats. Similarly, the blood glucose levels of the SDT fatty rats were significantly higher than those of the SD rats throughout the observation 
Kidney

Blood Pressure

Research
Kidney Blood Press Res 2019;44:1476-1492

DOI: 10.1159/000503926

(C) 2019 The Author(s).
www.karger.com/kbr

Tanabe et al.: Urinary L-FABP and Renal Hypoxia

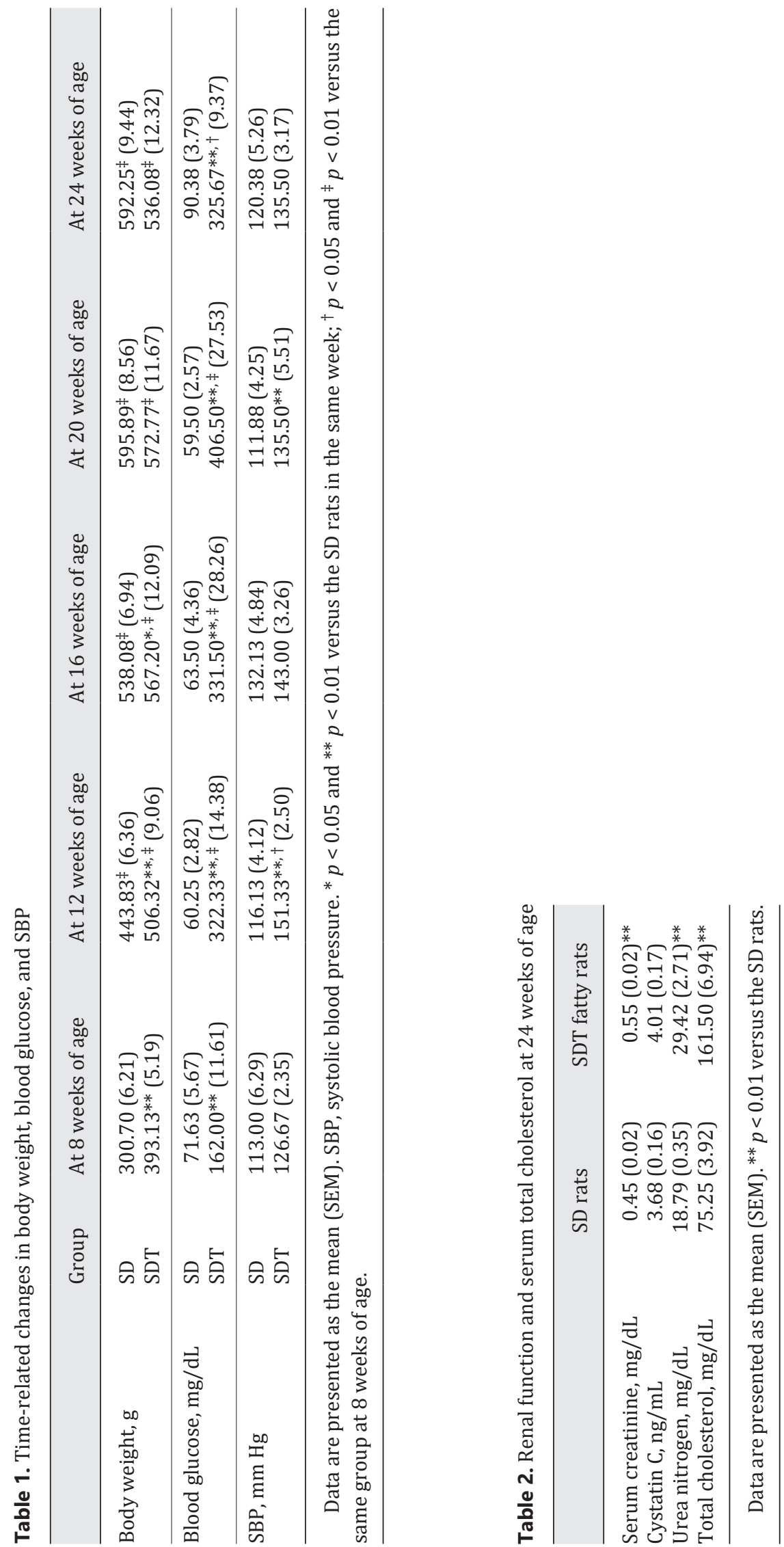


A

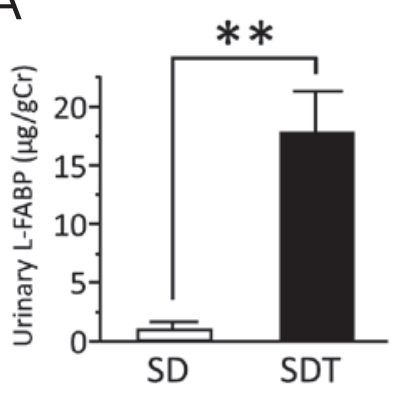

C

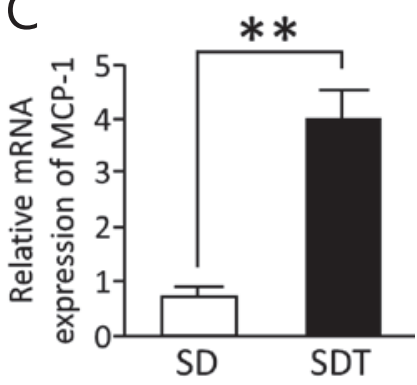

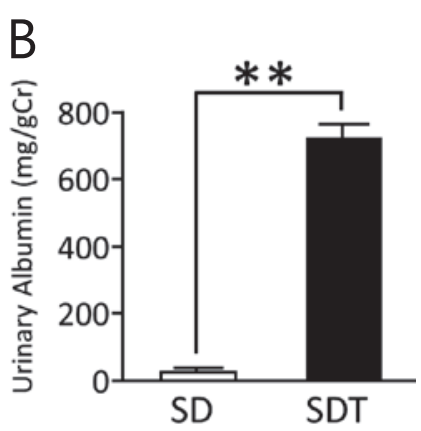

D

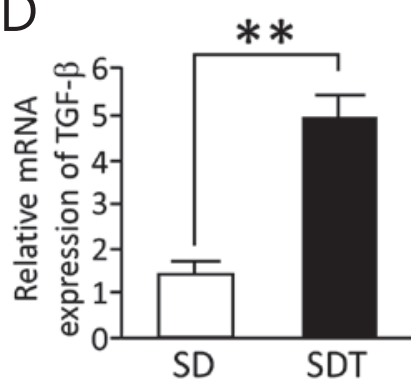

$\mathrm{E}$

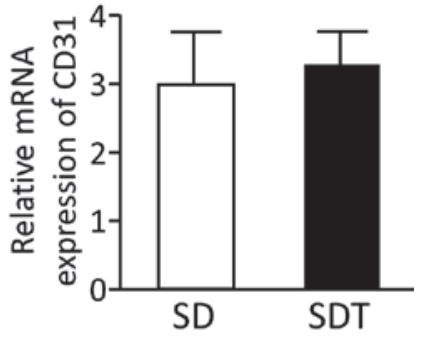

Fig. 1. Comparison of urinary parameters and renal mRNA transcript levels in Sprague-Dawley (SD) and spontaneously diabetic Torii (SDT) fatty rats at 24 weeks of age. A Urinary liver-type fatty acid-binding protein (L-FABP) levels at 24 weeks of age. B Urinary albumin at 24 weeks of age. mRNA transcript levels of monocyte chemoattractant protein $(M c p-1)(\mathbf{C})$, transforming growth factor $(T g f-\beta)(\mathbf{D})$, and $C D 31$ (E) in the kidney. Values are presented as means \pm standard error of the mean (SEM). ${ }^{* *} p<0.01$.

period. Although the SBP levels did not change over time in the SD rats, they were significantly higher at 12 weeks than at 8 weeks in the SDT fatty rats. Furthermore, they were significantly higher in the SDT fatty rats than in the SD rats at both 12 and 20 weeks.

\section{Comparison of Kidney Function and Total Cholesterol}

The SDT fatty rats exhibited significant increases in the levels of serum creatinine and urea nitrogen compared to the SD rats at 24 weeks of age (Table 2), but there was no significant difference in the serum cystatin $\mathrm{C}$ levels between the 2 groups. Conversely, the serum total cholesterol level in the SDT fatty rats was significantly higher than in the SD rats at 24 weeks of age.

\section{Comparison of Urinary Glucose, L-FABP, and Albumin Levels}

The mean scores for urinary glucose were 4 and 0 in the SDT fatty rats and SD rats, respectively, at 24 weeks of age. The levels of both urinary L-FABP and albumin were significantly higher in the SDT fatty rats than in the SD rats at 24 weeks of age (Fig. 1A, B).

\section{Increased Inflammatory Response and Profibrotic Activity in the SDT Fatty Rat Model of}

$D K D$

The mRNA levels of $M c p-1, T g f-\beta$, and $C D 31$ were measured to evaluate the inflammatory response, profibrotic activity, and presence of endothelial cells, respectively. The $M c p-1$ (Fig. 1C) and Tgf- $\beta$ (Fig. 1D) levels were significantly higher in the SDT fatty rats than in the SD rats, whereas the level of $C D 31$ was comparable between the SDT fatty rats and SD rats (Fig. 1E). 


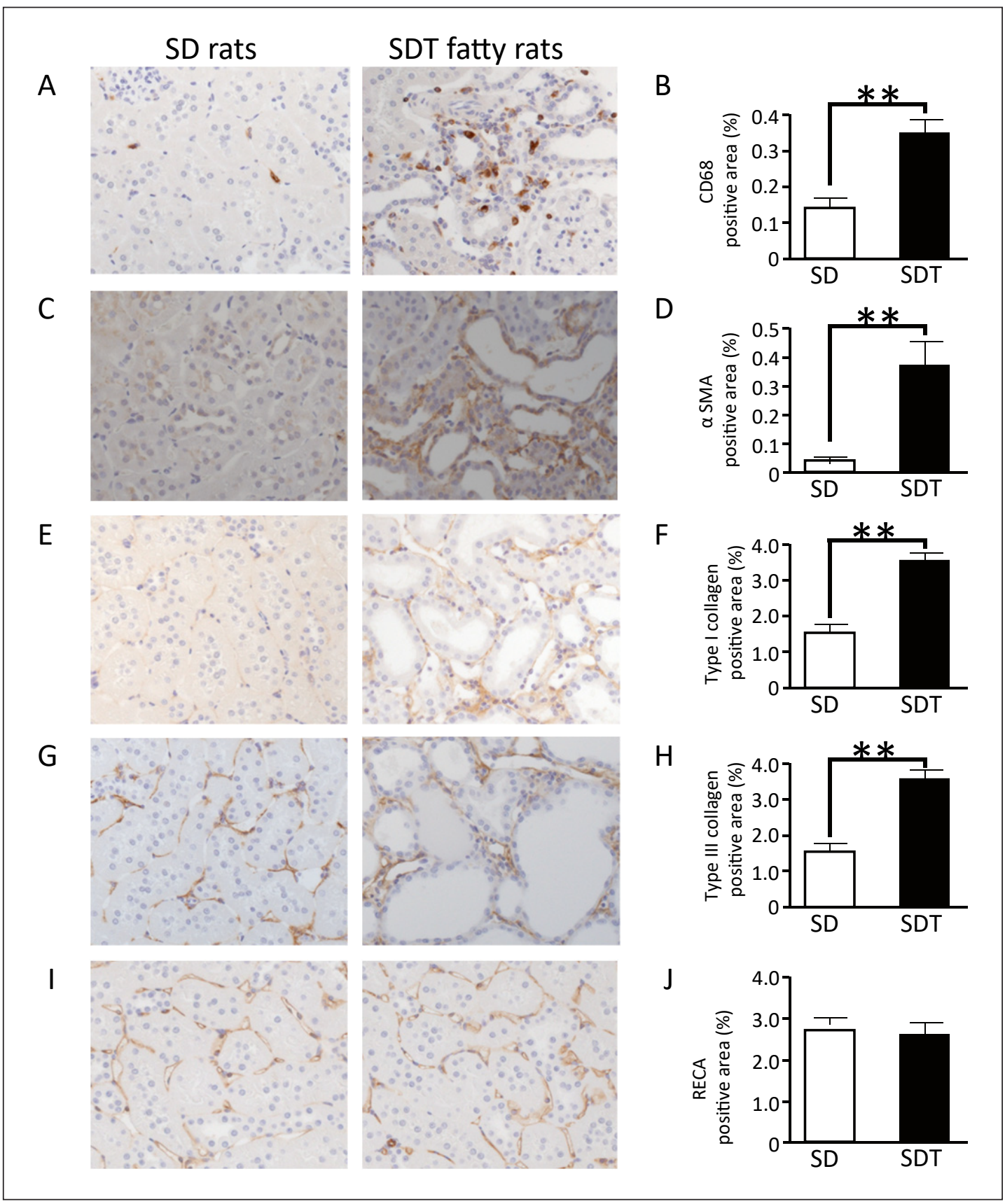

Fig. 2. Immunohistological staining using antibodies against CD68 (A, B), $\alpha$-smooth muscle actin $(\alpha$-SMA) (C, D), type I collagen (E, F), type III collagen $(\mathbf{G}, \mathbf{H})$, and rat endothelial cell antigen-1 (RECA-1) (I, J). $\times 100$. Values are presented as means \pm SEM. ${ }^{* *} p<0.01$.

\section{IHC Analysis of the Kidneys}

To evaluate the degree of renal interstitial inflammation, we utilized the macrophage marker CD68 in IHC analysis. The macrophages were detected in the interstitium of the SDT fatty rats (Fig. 2A), and the degree of macrophage infiltration was significantly greater than in the SD rats (Fig. 2B). 
Fig. 3. Histological staining with periodic acid-Schiff showing focal glomerular sclerosis (A) and semiquantitative assessment of focal glomerular sclerosis (B). IHC staining using an antibody against type IV collagen in the glomerulus (C, D). $\times 100$. Values are presented as means \pm SEM. $* p<0.01$

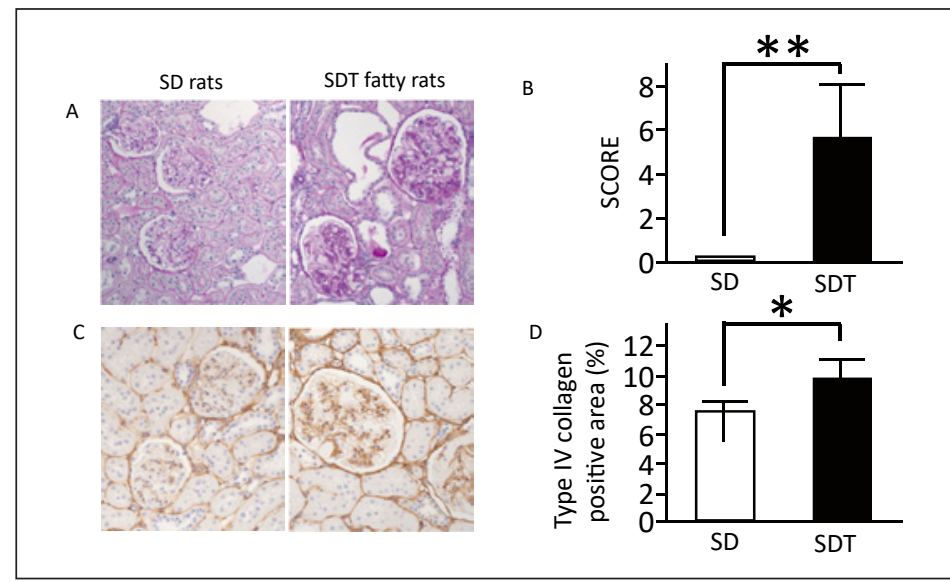

IHC analyses were also performed to evaluate tubulointerstitial fibrosis based on the expression levels of $\alpha$-SMA and type I and type III collagens. In the SDT fatty rats, $\alpha$-SMApositive areas were observed in the tubules and the interstitium (Fig. 2C); the extent of $\alpha$-SMA positivity was significantly greater in the SDT fatty rats than in the SD rats (Fig. 2D). Further analysis of the expression levels for type I (Fig. 2E) and type III (Fig. 2G) collagen in the interstitium revealed that the extent of positivity for both type I (Fig. 2F) and type III (Fig. 2H) collagen was significantly greater in the SDT fatty rats than in the SD rats.

Assessment of the number of endothelial cells in the peritubular capillaries by IHC analysis for RECA-1 revealed that the extent of RECA-1 positivity was comparable between the SDT fatty rats and the SD rats (Fig. 2I, J).

\section{Evaluation of Glomerular Sclerosis}

Focal glomerular sclerosis was observed in the PAS-stained kidney tissues of the SDT fatty rats (Fig. 3A), and the score for glomerular sclerosis was significantly greater than in the SD rats (Fig. 3B). Additionally, type IV collagen was immunolocalized to the mesangial areas in the glomeruli (Fig. 3C). Furthermore, the glomerular area that was positively stained for type IV collagen was significantly increased in the SDT fatty rats compared to the SD rats (Fig. 3D).

\section{Correlation between Urinary L-FABP Level and Histological Changes}

The urinary L-FABP levels correlated significantly with both the degree of macrophage infiltration determined by the CD68-positive areas (Fig. 4A), and tubulointerstitial fibrosis determined by the areas positive for $\alpha$-SMA (Fig. 4B) and type I (Fig. 4C) and type III (Fig. 4D) collagen.

\section{Analysis of HIF-1 $\alpha$ Expression and Correlation of HIF-1 $\alpha$ Protein Expression with Urinary}

\section{L-FABP Levels and Histological Changes}

The measurement of HIF- $1 \alpha$ expression levels in the kidneys to assess renal hypoxia revealed that both the mRNA (Fig. 5A) and protein (Fig. 5B) levels were significantly higher in the SDT fatty rats than in the SD rats.

The protein expression levels of HIF-1 $\alpha$ correlated significantly with the urinary L-FABP levels (Fig. 6A), macrophage infiltration determined by the CD68-positive areas (Fig. 6B), and tubulointerstitial fibrosis determined by the areas positive for $\alpha$-SMA (Fig. 6C) and type I 
A

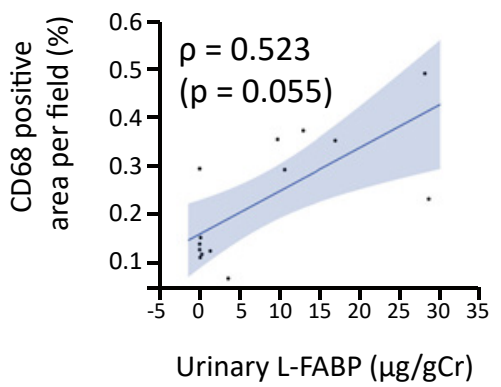

C

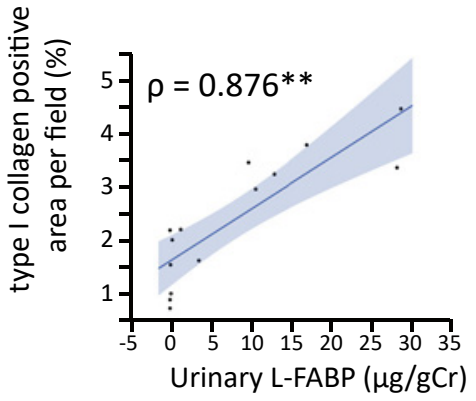

B

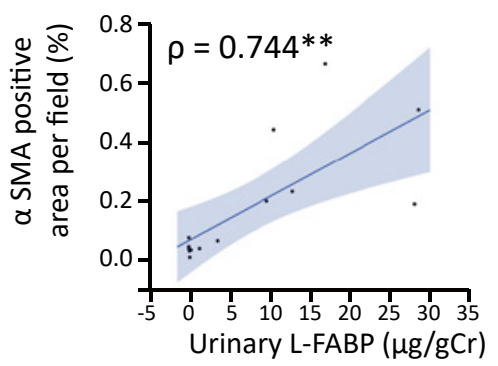

D

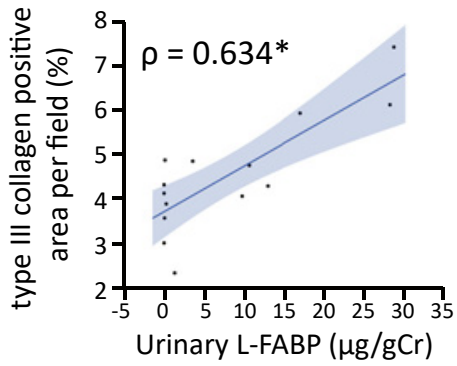

Fig. 4. Correlation between urinary L-FABP and the indicated renal tubulointerstitial damage markers. ${ }^{*} p<0.05,{ }^{* *} p<0.01$.

Fig. 5. Evaluation of renal hypoxia in the kidney. Gene expression (A) and protein expression (B) of hypoxia-inducible factor $1 \alpha$ (HIF$1 \alpha)$. Values are presented as means \pm SEM. $* p<0.05, * * p<$ 0.01 .

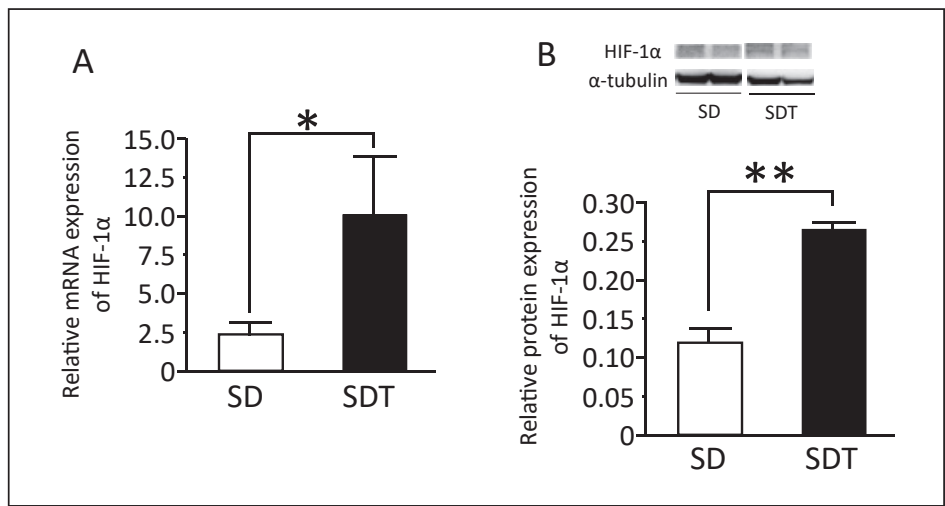

collagen (Fig. 6D). However, no similar correlation was observed between the protein expression levels of HIF- $1 \alpha$ and type III collagen (Fig. 6E).

Evaluation of Renal Blood Flow Using DCS and Correlation of Renal BFI with Urinary L-FABP Levels, Histological Changes, and Renal HIF-1 $\alpha$ Protein Expression

The analysis of the kidneys with DCS revealed that the microvascular BFI in the SDT fatty rats was significantly lower than in the SD rats (Fig. 7). 
A

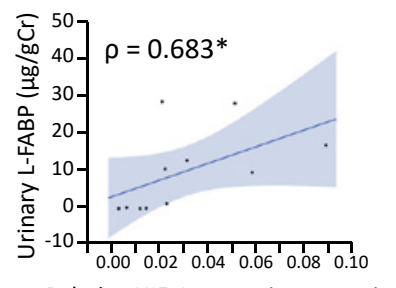

Relative HIF-1 $\alpha$ protein expression

D

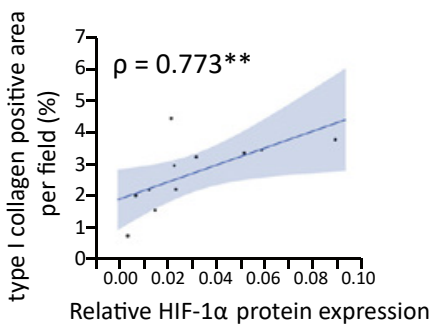

B

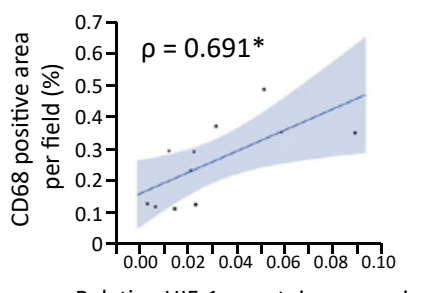

Relative HIF-1 $\alpha$ protein expression
C

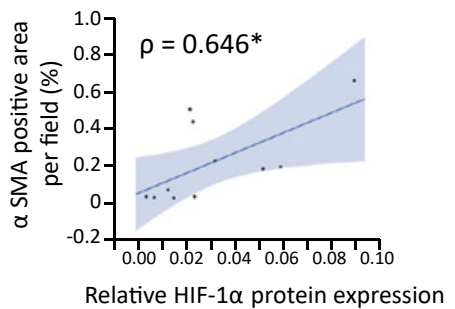

E

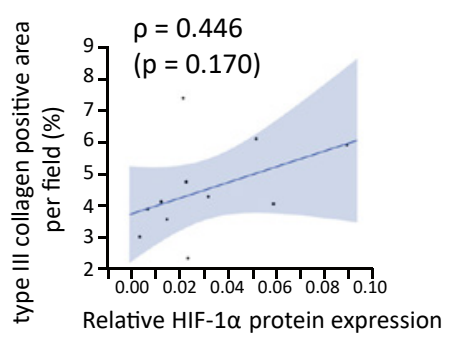

Fig. 6. Correlation between protein expression levels of HIF-1 $\alpha$ and urinary L-FABP $(\mathbf{A})$ and the indicated renal tubulointerstitial damage markers (B-E). ${ }^{*} p<0.05,{ }^{* *} p<0.01$.

Fig. 7. Comparison of blood flow index (BFI) between the SD and SDT fatty rats. Values are presented as means \pm SEM. $* p<0.05$.

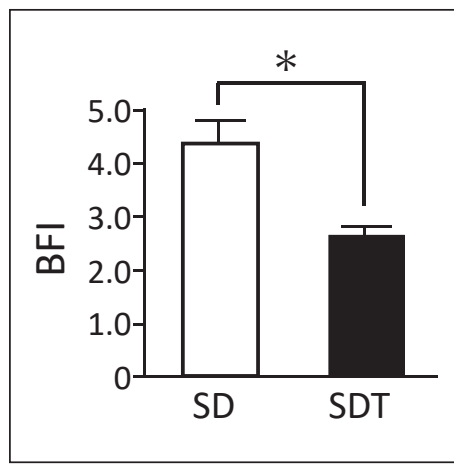

The renal BFI correlated significantly with the urinary L-FABP levels (Fig. 8A), tubulointerstitial fibrosis determined by the area positive for type I collagen (Fig. 8D), and renal protein expression levels of HIF-1 $\alpha$ (Fig. 8F). However, no such correlations were observed between the renal BFI and $\alpha$-SMA (Fig. 8C), or between the renal BFI and type III collagen (Fig. 8E). Conversely, the BFI tended to correlate with the macrophage infiltration determined by the CD68-positive areas (Fig. 8B).

\section{Analyses for eNOS Expression and Phosphorylation}

The endothelial function in the kidneys was determined by measuring the protein expression and phosphorylation levels of eNOS. The results indicated that the protein 


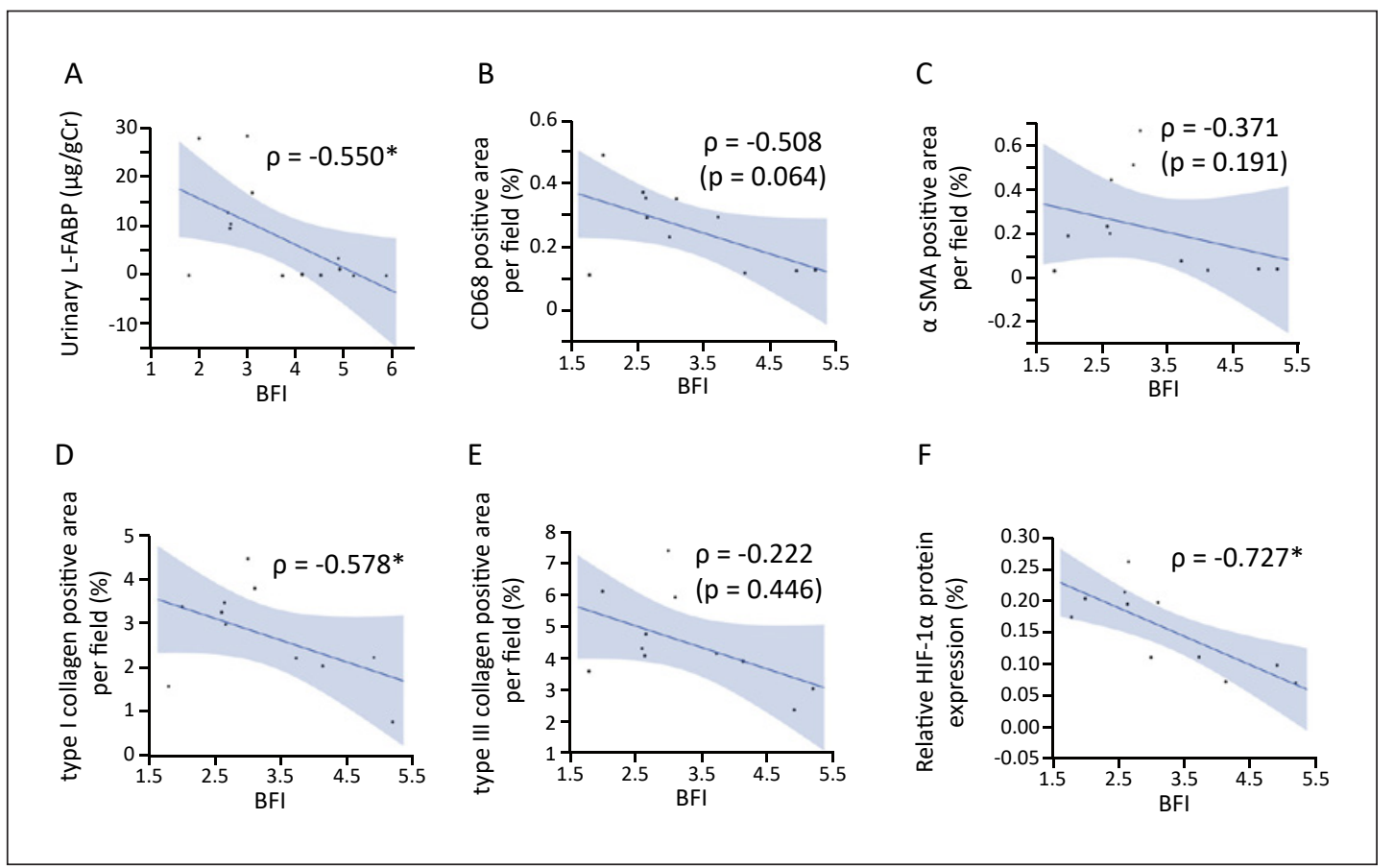

Fig. 8. Correlation between BFI and urinary L-FABP $(\mathbf{A})$, the indicated renal tubulointerstitial damage markers (B-E), and renal protein expression levels of HIF-1 $\alpha$ (F). ${ }^{*} p<0.05$.

expression levels of eNOS were significantly higher in the kidneys of the SDT fatty rats than in the SD rats (Fig. 9A, B), and that the phosphorylation (Fig. 9A, C) levels of eNOS were comparable between the SDT fatty rats and SD rats. Phosphorylated protein amount of eNOS was therefore considered to be higher in the kidneys of the SDT fatty rats than in the SD rats.

\section{Analysis of VEGF Expression}

The angiogenic activity in response to renal hypoxia was assessed by the measurement of VEGF expression levels in the kidneys, which revealed that both the mRNA (Fig. 9D) and protein (Fig. 9E) levels of VEGF were comparable between the SDT fatty rats and SD rats.

\section{Discussion}

Utilizing SDT fatty rats as a novel animal model of type 2 diabetes with obesity, we revealed that, compared to the control SD rats, the renal HIF- $1 \alpha$ levels were elevated and renal BFI was reduced in the SDT fatty rats, accompanied by renal tissue damage including glomerulosclerosis, interstitial inflammation, and fibrosis. The study also revealed a comparable frequency of endothelial cells in the peritubular capillaries, eNOS phosphorylation, and VEGF expression between the SDT fatty rats and the SD rats. These results suggest that the kidneys in the SDT fatty rats were under hypoxic conditions. In addition, the urinary L-FABP levels, which were significantly higher in the SDT fatty rats than in the SD rats, were positively correlated with the degree of renal HIF-1 $\alpha$ expression and negatively correlated with the renal BFI, suggesting that urinary L-FABP might reflect the degree of renal hypoxia in DKD. Along with previous evidence, the observed correlation of the renal expression of HIF-1 $\alpha$ and renal BFI with interstitial inflammation and interstitial fibrosis (Fig. 6, 8) provides support 


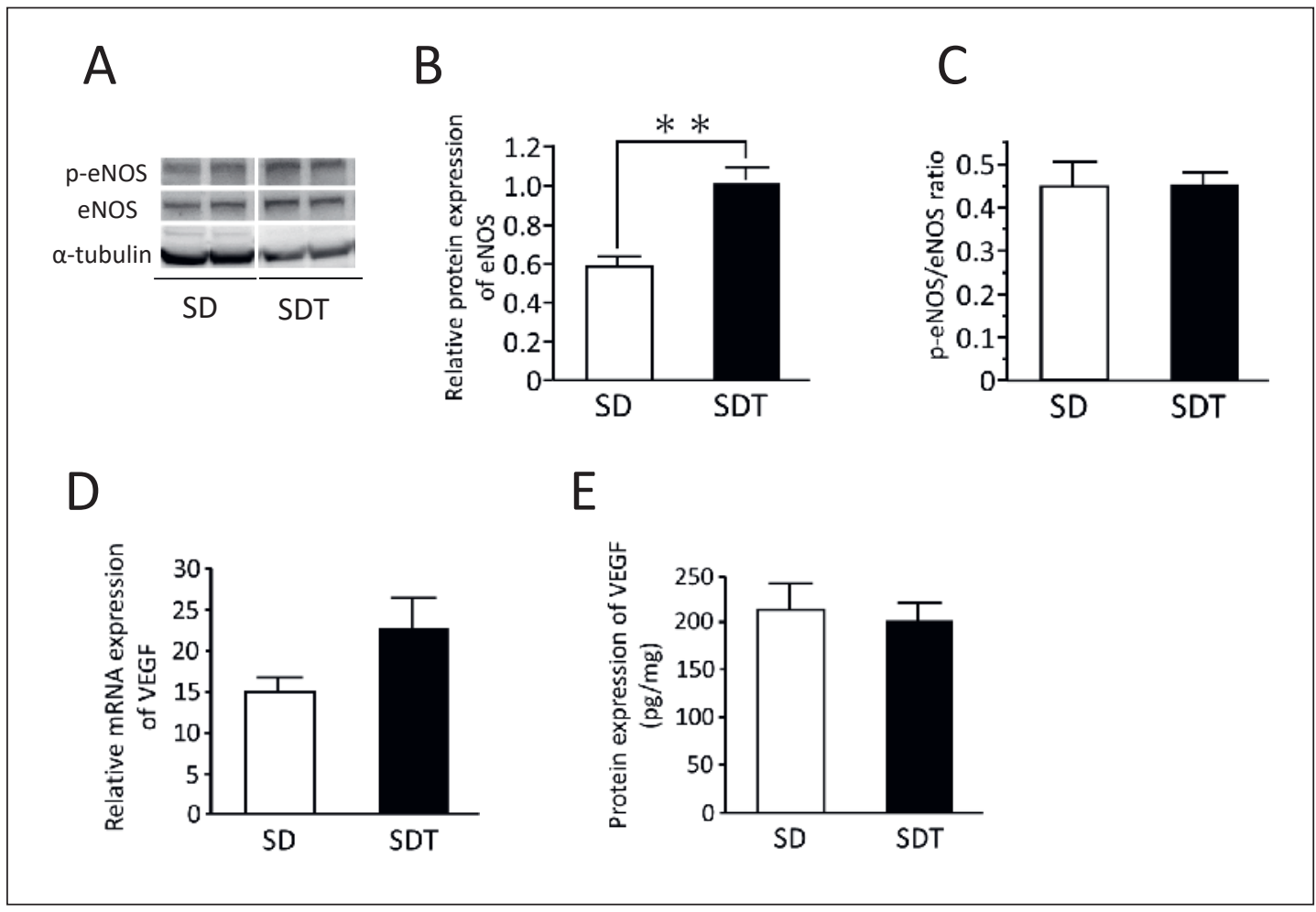

Fig. 9. Evaluations of endothelial nitric oxide synthase (eNOS) protein expression, phosphorylation of eNOS, and vascular endothelial growth factor (VEGF) gene and protein expressions in the kidney. Representative immunoblots (A), and quantitative evaluation of the protein expression (B) and phosphorylation (C) of eNOS. Gene (D) and protein expression (E) levels of VEGF. Values are presented as means \pm SEM. ${ }^{* *} p<0.01$.

for the notion that renal hypoxia is a crucial aggravating factor for tubulointerstitial damage in DKD $[7,8]$. These results further suggest that, by detecting renal hypoxia, urinary L-FABP might be useful for monitoring DKD in clinical practice. Although urinary L-FABP was reported to be associated with renal microvascular blood flow in renal transplantation [19], our study is the first to report its potential as a renal hypoxic marker in DKD in type 2 diabetes.

SDT fatty rats, utilized in numerous diabetic animal models, exhibit various metabolic complications frequently observed in patients with type 2 diabetes, such as obesity, hyperlipidemia, and hypertension [20]. In patients with type 2 diabetes, various metabolic abnormalities have an impact on the kidneys, in addition to hyperglycemia, and numerous factors contribute to the onset and/or progression of kidney disease. The renal pathophysiology in SDT fatty rats may mimic the DKD observed in type 2 diabetic patients with numerous metabolic complications. Both macroalbuminuria and mild renal dysfunction were observed in the 24-week-old SDT fatty rats. Our results may thus reflect the relationship between renal hypoxia and urinary L-FABP levels in DKD with macroalbuminuria observed in humans.

Two mechanisms underlie the induction of renal hypoxia in type 2 diabetes: elevated renal oxygen consumption and changes in renal hemodynamics. One study reported a $>40 \%$ increase in oxygen consumption in the renal tubules of diabetic rats compared to nondiabetic rats [29]. The increase in renal oxygen consumption is attributed to increased renal tubular reabsorption of massive levels of glucose filtered in the glomeruli as a result of hyperglycemia, increased electrolyte levels after glomerular hyperfiltration [7, 30], and increased oxidative stress in diabetes [31]. Conversely, renal hemodynamics are altered by both 


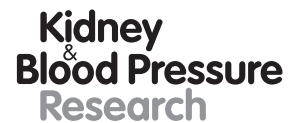

Kidney
Blood Pressure

Research \begin{tabular}{l|l}
\hline Kidney Blood Press Res 2019;44:1476-1492 \\
\hline DOI: 10.1159/000503926 & $\begin{array}{l}\text { @ 2019 The Author(s). Published by S. Karger AG, Basel } \\
\text { www.karger.com/kbr }\end{array}$ \\
\hline
\end{tabular}

Tanabe et al.: Urinary L-FABP and Renal Hypoxia

glomerular and tubular damage in addition to a decrease in whole renal blood flow due to arteriosclerosis. Glomerular damage, which is induced by hyperglycemia, obesity, hypertension, and arteriolosclerosis, leads to a decrease in postglomerular blood flow, resulting in tubular hypoxia. Furthermore, a direct insult to the tubular cells by hyperglycemia leads to the production of inflammatory cytokines which provoke peritubular capillary damage [32, 33], resulting in the induction of tubular hypoxia [34]. In this study, there was an increase in urinary glucose excretion in the SDT fatty rats. Furthermore, while we failed to observe a decrease in endothelial cell numbers or eNOS activation, we found an increase in renal HIF-1 $\alpha$ expression, a reduction in renal BFI, and mild glomerulosclerosis in the SDT fatty rats. Therefore, we attribute the renal hypoxia observed in 24-week-old SDT fatty rats to elevated renal oxygen consumption that was due to the increased renal tubular absorption of glucose as well as a reduction in glomerular and postglomerular blood flow as a result of glomerulosclerosis.

Adaptation of the kidneys to renal hypoxia occurs via an increase in HIF-1 $\alpha$ expression, which leads to the upregulation of VEGF that accelerates angiogenesis [35-37]. Our results did not reveal an increase in the renal expression of VEGF in the SDT fatty rats compared to the SD rats, but the renal HIF-1 $\alpha$ expression was significantly increased in the SDT fatty rats.

It has been reported that hyperglycemia suppresses angiogenesis despite the increased gene expression of VEGF receptors in cocultures of human proximal tubular cells and endothelial cells [36]. Furthermore, hyperglycemia during hypoxia was found to inhibit the upregulation of VEGF expression due to oxidative stress in rat proximal tubular cells [38]. Our results lend further support to these previous in vitro findings.

We used DCS to measure renal microvascular blood flow. The currently adopted interval between photon-emitter and detector probes is $4 \mathrm{~mm}$, with which the microvascular blood flow of the corticomedullary junction approximately $2 \mathrm{~mm}$ below the renal surface can be detected. The corticomedullary junction is reported to consume approximately half the oxygen of the whole kidney due to the wide distribution of the electrolytic pumps using ATP [39]; this area is therefore particularly vulnerable to hypoxia. Conversely, the corticomedullary junction is susceptible to hypoxia in kidney disease because the blood flow in this area is solely dependent on the postglomerular flow of the juxtamedullary glomeruli. Specifically, hypertension, a major and frequent complication of type 2 diabetes [40], initially induces vascular damage in the juxtamedullary glomeruli, and this leads to hypoxia in the corticomedullary and medullary regions by impairing renal circulation [41]. Therefore, the DCS method for measuring microvascular blood flow in the corticomedullary region utilized here was suitable for detecting renal hypoxia.

The results of this study may have been limited by several factors. First, the presence of anemia was not evaluated. There is a possibility that renal anemia occurs in SDT fatty rats with mild renal function. As the DCS method detects the movement of red blood cells, changes in the number of these cells might affect BFI measurement. The potential confounding effect of anemia should be elucidated in future studies. Second, the correlation between renal hypoxia and DKD progression was not investigated. The progression of DKD was mild, even in the SDT fatty rats with macroalbuminuria $[42,43]$; therefore, older SDT fatty rats, such as 60 -week-old animals, should be evaluated for the correlation of renal hypoxia with the progression to advanced DKD [44]. One of the SDT fatty rats died at 23 weeks of age and the presumed cause was malnutrition due to the long-term impairment of glucose utilization, although necropsy was not performed. So no long-term assessment was conducted. Third, because of the small sample size in the basic research, we did not determine the most suitable marker to accurately reflect the degree of renal hypoxia. More clinical research is needed to reveal the point. Finally, we did not investigate changes in urinary L-FABP levels in response to interventions that ameliorate renal hypoxia. A recent study demonstrated that habitual 
exercise leads to a reduction in urinary L-FABP levels via a decrease in renovascular resistance and an improvement of renal microcirculation in healthy middle-aged and older adults [45]. Another clinical study reported that urinary L-FABP decreased along with the improvement of renal disease due to various treatments [46]. Urinary L-FABP may thus respond to the elimination of renal hypoxia.

In conclusion, we revealed that urinary L-FABP levels reflect the degree of renal hypoxia, as evaluated by renal HIF- $1 \alpha$ expression and BFI measured with DCS in DKD using a type 2 diabetic animal model. Although further investigation is necessary, these findings suggest that urinary L-FABP might be useful as a renal hypoxic marker to monitor DKD in patients with type 2 diabetes in clinical practice because renal hypoxia is an aggravating factor for the progression of DKD.

\section{Acknowledgments}

We thank Ms. Kimie Katayama and Ms. Junko Asano for the technical assistance.

\section{Disclosure Statement}

T.S. is the director and a senior scientist at CMIC Holdings Co., Ltd., and K.O. is a scientist at CMIC Holdings Co., Ltd., which produced the kits for L-FABP analysis. All other authors declare no conflicts of interest.

\section{Funding Sources}

This research did not receive any specific grant from funding agencies in the public, commercial, or not-for-profit sectors.

\section{Author Contributions}

J.T., T.S., K.O., and A.K.-I.: study design. J.T., Y.O., M.N., K.O., K.K., S.W., K.I., S.H., Y.O., and A.K.-I.: data collection. J.T. and A.K.-I.: data analysis, literature search, and the generation of the figures. J.T., Y.N., D.I., T.S., K.O., K.K., Y.S., and A.K.-I.: data interpretation. J.T., Y.S., and A.K.-I.: writing of the manuscript. All authors provided final approval of the submitted version.

\section{References}

1 Afkarian M, Zelnick LR, Hall YN, Heagerty PJ, Tuttle K, Weiss NS, et al. Clinical manifestations of kidney disease among US adults with diabetes, 1988-2014. JAMA. 2016 Aug;316(6):602-10.

2 Doshi SM, Friedman AN. Diagnosis and Management of Type 2 Diabetic Kidney Disease. Clin J Am Soc Nephrol. 2017 Aug;12(8):1366-73.

3 Tuttle KR, Bakris GL, Bilous RW, Chiang JL, de Boer IH, Goldstein-Fuchs J, et al. Diabetic kidney disease: a report from an ADA Consensus Conference. Am J Kidney Dis. 2014 Oct;64(4):510-33.

4 Mise K, Hoshino J, Ueno T, Hazue R, Hasegawa J, Sekine A, et al. Prognostic Value of Tubulointerstitial Lesions, Urinary $\mathrm{N}$-acetyl- $\beta$-d-glucosaminidase, and urinary $\beta 2$-microglobulin in patients with type 2 diabetes and biopsy-proven diabetic nephropathy. Clin J Am Soc Nephrol. 2016 Apr;11(4):593-601.

5 Mise K, Hoshino J, Ueno T, Hazue R, Sumida K, Hiramatsu R, et al. Clinical and pathological predictors of estimated GFR decline in patients with type 2 diabetes and overt proteinuric diabetic nephropathy. Diabetes Metab Res Rev. 2015 Sep;31(6):572-81. 
6 Shimizu M, Furuichi K, Toyama T, Kitajima S, Hara A, Kitagawa K, et al.; Kanazawa Study Group for Renal Diseases and Hypertension. Long-term outcomes of Japanese type 2 diabetic patients with biopsy-proven diabetic nephropathy. Diabetes Care. 2013 Nov;36(11):3655-62.

7 Friederich-Persson M, Thörn E, Hansell P, Nangaku M, Levin M, Palm F. Kidney hypoxia, attributable to increased oxygen consumption, induces nephropathy independently of hyperglycemia and oxidative stress. Hypertension. 2013 Nov;62(5):914-9.

8 Hansell P, Welch WJ, Blantz RC, Palm F. Determinants of kidney oxygen consumption and their relationship to tissue oxygen tension in diabetes and hypertension. Clin Exp Pharmacol Physiol. 2013 Feb;40(2):123-37.

9 Nangaku M. Chronic hypoxia and tubulointerstitial injury: a final common pathway to end-stage renal failure. J Am Soc Nephrol. 2006 Jan;17(1):17-25.

10 Sasaki H, Kamijo-Ikemori A, Sugaya T, Yamashita K, Yokoyama T, Koike J, et al. Urinary fatty acids and livertype fatty acid binding protein in diabetic nephropathy. Nephron Clin Pract. 2009;112(3):c148-56.

11 Watanabe S, Ichikawa D, Sugaya T, Ohata K, Inoue K, Hoshino S, et al. Urinary Level of Liver-Type Fatty Acid Binding Protein Reflects the Degree of Tubulointerstitial Damage in Polycystic Kidney Disease. Kidney Blood Press Res. 2018;43(6):1716-29.

12 Yokoyama T, Kamijo-Ikemori A, Sugaya T, Hoshino S, Yasuda T, Kimura K. Urinary excretion of liver type fatty acid binding protein accurately reflects the degree of tubulointerstitial damage. Am J Pathol. 2009 Jun; 174(6): 2096-106.

13 Hisamichi M, Kamijo-Ikemori A, Sugaya T, Ichikawa D, Hoshino S, Hirata K, et al. Increase in urinary markers during the acute phase reflects the degree of chronic tubulointerstitial injury after ischemia-reperfusion renal injury. Biomarkers. 2017 Feb;22(1):5-13.

14 Ichikawa D, Kamijo-Ikemori A, Sugaya T, Ohata K, Hisamichi M, Hoshino S, et al. Utility of urinary tubular markers for monitoring chronic tubulointerstitial injury after ischemia-reperfusion. Nephrology (Carlton). 2018 Apr;23(4):308-16.

15 Matsui K, Kamijo-Ikemori A, Imai N, Sugaya T, Yasuda T, Tatsunami S, et al. Clinical significance of urinary liver-type fatty acid-binding protein as a predictor of ESRD and CVD in patients with CKD. Clin Exp Nephrol. 2016 Apr;20(2):195-203.

16 Kamijo-Ikemori A, Sugaya T, Yasuda T, Kawata T, Ota A, Tatsunami S, et al. Clinical significance of urinary livertype fatty acid-binding protein in diabetic nephropathy of type 2 diabetic patients. Diabetes Care. 2011 Mar; 34(3):691-6.

17 Panduru NM, Forsblom C, Saraheimo M, Thorn L, Bierhaus A, Humpert PM, et al.; FinnDiane Study Group. Urinary liver-type fatty acid-binding protein and progression of diabetic nephropathy in type 1 diabetes. Diabetes Care. 2013 Jul;36(7):2077-83.

18 Colhoun HM, Marcovecchio ML. Biomarkers of diabetic kidney disease. Diabetologia. 2018 May;61(5):9961011.

19 Yamamoto T, Noiri E, Ono Y, Doi K, Negishi K, Kamijo A, et al. Renal L-type fatty acid-binding protein in acute ischemic injury. J Am Soc Nephrol. 2007 Nov;18(11):2894-902.

20 Matsui K, Ohta T, Oda T, Sasase T, Ueda N, Miyajima K, et al. Diabetes-associated complications in spontaneously diabetic Torii fatty rats. Exp Anim. 2008 Apr;57(2):111-21.

21 Durduran T, Choe R, Baker WB, Yodh AG. Diffuse Optics for Tissue Monitoring and Tomography. Rep Prog Phys. 2010 Jul;73(7):73.

22 Ichinose M, Nakabayashi M, Ono Y. Sympathoexcitation constrains vasodilation in the human skeletal muscle microvasculature during postocclusive reactive hyperemia. Am J Physiol Heart Circ Physiol. 2018 Aug; 315(2):H242-53.

23 Ichinose M, Nakabayashi M, Ono Y. Difference in the integrated effects of sympathetic vasoconstriction and local vasodilation in human skeletal muscle and skin microvasculature. Physiol Rep. 2019 Apr;7(7):e14070.

24 Yu G, Durduran T, Lech G, Zhou C, Chance B, Mohler ER 3rd, et al. Time-dependent blood flow and oxygenation in human skeletal muscles measured with noninvasive near-infrared diffuse optical spectroscopies. J Biomed Opt. 2005 Mar-Apr;10(2):024027.

25 Ichikawa D, Kamijo-Ikemori A, Sugaya T, Yasuda T, Hoshino S, Igarashi-Migitaka J, et al. Renal liver-type fatty acid binding protein attenuates angiotensin II-induced renal injury. Hypertension. 2012 Oct;60(4):973-80.

26 Hisamichi M, Kamijo-Ikemori A, Sugaya T, Ichikawa D, Natsuki T, Hoshino S, et al. Role of angiotensin II type 1a receptor in renal injury induced by deoxycorticosterone acetate-salt hypertension. FASEB J. 2017 Jan; 31(1):72-84.

27 Hisamichi M, Kamijo-Ikemori A, Sugaya T, Hoshino S, Kimura K, Shibagaki Y. Role of bardoxolone methyl, a nuclear factor erythroid 2-related factor 2 activator, in aldosterone- and salt-induced renal injury. Hypertens Res. 2018 Jan;41(1):8-17.

28 Ohata K, Kamijo-Ikemori A, Sugaya T, Hibi C, Nakamura T, Murase T, et al. Renoprotective effect of the xanthine oxidoreductase inhibitor Topiroxostat under decreased angiotensin II type 1a receptor expression. Eur J Pharmacol. 2017 Nov;815:88-97.

29 Ries M, Basseau F, Tyndal B, Jones R, Deminière C, Catargi B, et al. Renal diffusion and BOLD MRI in experimental diabetic nephropathy. Blood oxygen level-dependent. J Magn Reson Imaging. 2003 Jan;17(1):104-13.

30 Baines A, Ho P. Glucose stimulates $\mathrm{O} 2$ consumption, $\mathrm{NOS}$, and $\mathrm{Na} / \mathrm{H}$ exchange in diabetic rat proximal tubules. Am J Physiol Renal Physiol. 2002 Aug;283(2):F286-93. 
31 Palm F, Cederberg J, Hansell P, Liss P, Carlsson PO. Reactive oxygen species cause diabetes-induced decrease in renal oxygen tension. Diabetologia. 2003 Aug;46(8):1153-60.

32 Navarro-González JF, Mora-Fernández C, Muros de Fuentes M, García-Pérez J. Inflammatory molecules and pathways in the pathogenesis of diabetic nephropathy. Nat Rev Nephrol. 2011 Jun;7(6):327-40.

33 Mudaliar H, Pollock C, Komala MG, Chadban S, Wu H, Panchapakesan U. The role of Toll-like receptor proteins (TLR) 2 and 4 in mediating inflammation in proximal tubules. Am J Physiol Renal Physiol. 2013 Jul;305(2):F14354.

34 Gilbert RE. Proximal Tubulopathy: Prime Mover and Key Therapeutic Target in Diabetic Kidney Disease. Diabetes. 2017 Apr;66(4):791-800.

35 Shweiki D, Itin A, Soffer D, Keshet E. Vascular endothelial growth factor induced by hypoxia may mediate hypoxia-initiated angiogenesis. Nature. 1992 Oct;359(6398):843-5.

36 Kim BS, Chen J, Weinstein T, Noiri E, Goligorsky MS. VEGF expression in hypoxia and hyperglycemia: reciprocal effect on branching angiogenesis in epithelial-endothelial co-cultures. J Am Soc Nephrol. 2002 Aug;13(8): 2027-36.

37 Shu S, Wang Y, Zheng M, Liu Z, Cai J, Tang C, et al. Hypoxia and Hypoxia-Inducible Factors in Kidney Injury and Repair. Cells. 2019 Feb;8(3):8.

38 Katavetin P, Miyata T, Inagi R, Tanaka T, Sassa R, Ingelfinger JR, et al. High glucose blunts vascular endothelial growth factor response to hypoxia via the oxidative stress-regulated hypoxia-inducible factor/hypoxiaresponsible element pathway. J Am Soc Nephrol. 2006 May;17(5):1405-13.

39 Ochi M, Yoshioka H, Sawada T, Kusunoki T, Hattori T. New adipocyte formation in mice during refeeding after long-term deprivation. Am J Physiol. 1991 Mar;260(3 Pt 2):R468-74.

40 Tatsumi Y, Ohkubo T. Hypertension with diabetes mellitus: significance from an epidemiological perspective for Japanese. Hypertens Res. 2017 Sep;40(9):795-806.

41 Ito S, Nagasawa T, Abe M, Mori T. Strain vessel hypothesis: a viewpoint for linkage of albuminuria and cerebrocardiovascular risk. Hypertens Res. 2009 Feb;32(2):115-21.

42 Katsuda Y, Kemmochi Y, Maki M, Sano R, Ishii Y, Miyajima K, et al. Physiological changes induced by salt intake in female Spontaneously Diabetic Torii-Lepr(fa) (SDT fatty) rat, a novel obese type 2 diabetic model. Anim Sci J. 2014 May;85(5):588-94.

43 Katsuda Y, Kemmochi Y, Maki M, Sano R, Toriniwa Y, Ishii Y, et al. Effects of unilateral nephrectomy on renal function in male Spontaneously Diabetic Torii fatty rats: a novel obese type 2 diabetic model. J Diabetes Res. 2014;2014:363126.

44 Kemmochi Y, Fukui K, Maki M, Kimura S, Ishii Y, Sasase T, et al. Metabolic Disorders and Diabetic Complications in Spontaneously Diabetic Torii Lepr (fa) Rat: A New Obese Type 2 Diabetic Model. J Diabetes Res. 2013; 2013:948257.

45 Kosaki K, Kamijo-Ikemori A, Sugaya T, Tanahashi K, Sawano Y, Akazawa N, et al. Effect of habitual exercise on urinary liver-type fatty acid-binding protein levels in middle-aged and older adults. Scand J Med Sci Sports. 2018 Jan;28(1):152-60.

46 Kamijo-Ikemori A, Sugaya T, Ichikawa D, Hoshino S, Matsui K, Yokoyama T, et al. Urinary liver type fatty acid binding protein in diabetic nephropathy. Clin Chim Acta. 2013 Sep;424:104-8. 